\title{
PENALIZATION IN DENSITY-BASED TOPOLOGY OPTIMIZATION: A MATHEMATICAL PERSPECTIVE
}

\author{
MOHAMED ABDELHAMID ${ }^{1}$ AND ALEKSANDER CZEKANSKI ${ }^{2}$ \\ ${ }^{1}$ Department of Mechanical Engineering, York University \\ 4700 Keele St., Toronto, Ontario, M3J 1P3, Canada \\ mahamid@yoku.ca \\ 2 Department of Mechanical Engineering, York University \\ 4700 Keele St., Toronto, Ontario, M3J 1P3, Canada \\ alex.czekanski@lassonde.yorku.ca
}

Key words: Topology Optimization, Density-based Methods, Initial Guess, Local Minima, Penalization, Convexity

\begin{abstract}
Most real-life engineering optimization problems are non-convex by nature. In a topology optimization context, this non-convexity is even exacerbated by the extra restrictions imposed during the optimization process to enforce mesh-independent black/white manufacturable solutions. Such restrictions include intermediate density penalization, as well as external regulation techniques imposed to tackle some numerical instabilities such as checkerboarding and mesh dependence, in addition to various design constraints. This non-convexity gives rise to the problem of local minima, where the converged solution is greatly affected by the algorithmic parameters as well as the initial guess. To overcome this non-convexity, it's often advised to use continuation methods, that is to introduce non-convexification gradually between iterations. In this article, we present a comprehensive treatment of the sources of nonconvexity in density-based topology optimization problems, with a special emphasis on linear elastic compliance minimization. This is in an attempt to better bridge the gap between the mathematical and the engineering/physical aspects of the topic.
\end{abstract}

\section{INTRODUCTION}

Since the introduction of the Solid Isotropic Material with Penalization (SIMP) method in the seminal paper by Bendsøe [3], material interpolation methods have become one of the most active research areas in engineering optimization. Although the origin of almost all density-based approaches lies in linear elasticity, they have been successfully extended to even more complicated single and multiphysics fields. In a general sense, the optimal topology of a problem refers to the location and number of holes such that an objective function is extremized.

To simplify the numerical implementation of topology optimization formulations, the normalized density is usually taken to be element-wise constant rather than the less popular node-wise approach. This way the design variables can be taken outside the integral of the elemental stiffness matrices (c.f. Sigmund and Petersson [25, p. 68] and Petersson and Sigmund [21, p. 1419]). A discrete formulation, where the normalized density is only allowed to take discrete $0 / 1$ values, proves to be extremely difficult to solve 
numerically [27]. Hence relaxation of the design space is introduced, namely continuous ${ }^{1}$ variation of the design variables, where intermediate density elements between the void and material states are allowed to exist during the optimization process [5]. In order to obtain practical manufacturable designs, intermediate density elements have to be eliminated from the final design. Penalization, in different forms, is introduced to increase the cost (i.e. reduce the benefit) of intermediate elements to force the solution to converge towards discrete $0 / 1$ designs. The concept of continuous design variables with penalization constitutes the essence of material interpolation methods.

It is a widely known fact that any approach that enforces discrete $0 / 1$ solutions is inherently non-convex. A rather unfavorable consequence of this non-convexity is the obscureness of the global optimum. In other words, the converged solution of non-global optimization approaches would be one of the huge pool of local minima the problem possesses. This is mainly due to the fact that in a non-convex function, the global optimum can't be identified using certain mathematical conditions, as opposed to the local optimum that can be identified through the local behavior of the function, namely its gradient and Hessian. Hence, in a non-convex problem, it is impossible to calculate, or even claim, the global optimum without an exhaustive search of the entire design space [1, p. 138-139]. To complicate the problem further, global optimization approaches are extremely computationally extensive, and, so far, prove unable to handle the typically massive number of design variables in practical topology optimization problems [25, p. 74].

Even though the final desired solution in a topology optimization problem is ideally pure discrete with no intermediate density elements, the discrete form of the problem is almost impossible to challenge. Hence, in a discussion on global optimality, the logical path to follow is to start from the relaxed convex form of the compliance minimization problem that has an easily-identifiable
thickness sheet problem. Starting from this global optimum, we invest
convexity of the problem, how continuation methods can be used to
how the initial guess has some weight in determining the final optim 2 THE VARIABLE THICIKNESS SHEET PROBLEM

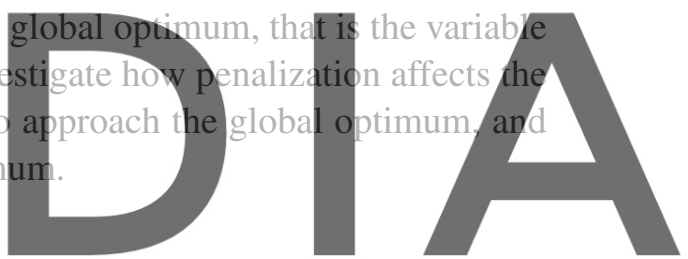

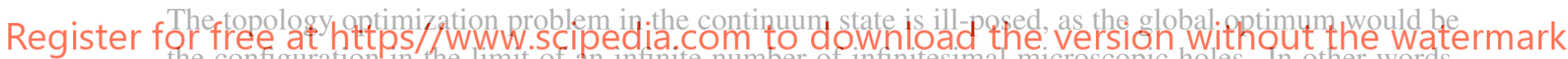
the configuration in the limit of an infinite number of infinitesimal microscopic holes. In other words, there is always a better global optimum by decreasing the size and increasing the number of these microscopic holes, which means the solution set is not closed. From a mathematical sense, the correct term to use would be a global infimum, not a minimum, meaning it can be approached but never attained.

In order to fully comprehend the effect of domain discretization on convexity, it's worthwhile to revisit the definition of convex functions. Bazaraa et al. [2, p. 98] define a function to be convex if a line segment drawn between any two points on its graph falls entirely on or above the graph. This definition has two implications; one on the domain of the function and another on its codomain. Considering first the domain (i.e. the set of solution points), it has to form a convex set. Meaning it has to be continuous and its boundaries must not curve into the set. As for the codomain, for a function $f(\mathbf{x})$ with $S$ being a nonempty set in $R^{N}$, convexity requires that the function's epigraph (i.e. the set of points on or above its graph) defined as:

\footnotetext{
${ }^{1}$ To avoid confusion, continuous is used to describe the opposite of discrete design variable, while continuum is used to describe the opposite of discretized geometrical spatial domains.
} 


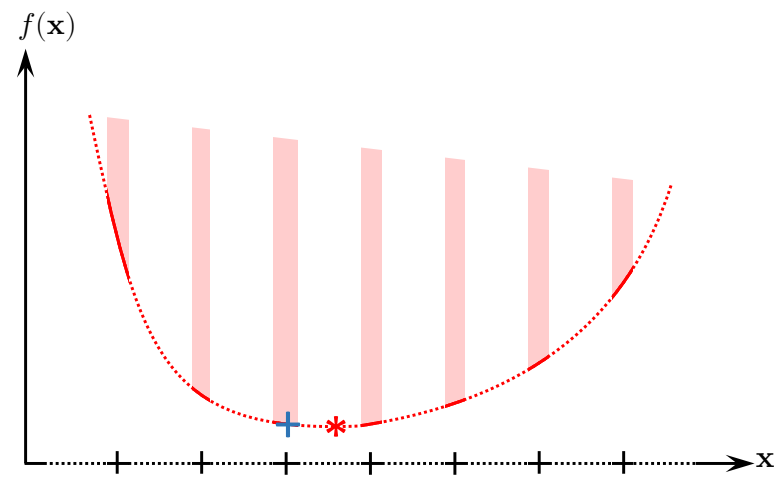

(a)

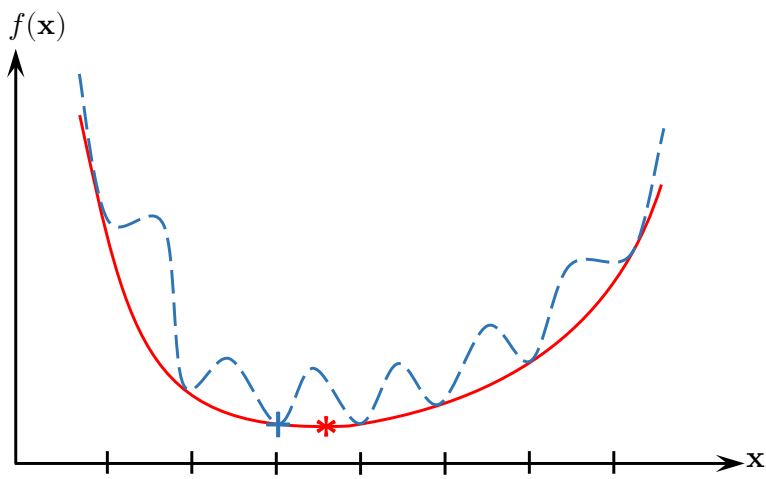

(b)

Figure 1: Non-convexification of a convex function by: a. Domain Discretization: the dotted lines represent the solution points removed due to domain discretization, while the light red areas represent the function's epigraph after discretization. b. Penalization: the original convex function is shown in continuous red, while the penalized non-convex version is shown in dashed blue. In both cases, the global minimum is marked before nonconvexification by a red asterisk, and after non-convexification by a blue plus symbol. The tick marks on the horizontal axis represent the desirable design points, whereas the undesirable points are represented by the values in between.

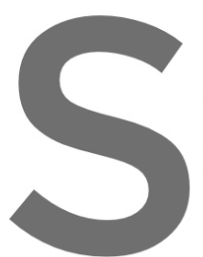

\[ \{(\mathbf{x}, y) ; \mathbf{x} \in S, \boldsymbol{y} \]
be a convex set itself. The first implication relates to
penalization.
The effect of transforming the continuum domain in
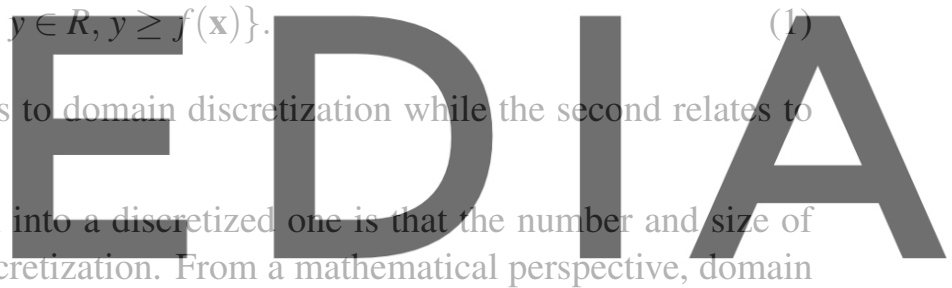

the potential holes is limited as a result of this discretization. From a mathematical perspective, domain
discretization ${ }^{2}$ causes non-convexity by "poking holes" in the original convex set of solution points, that

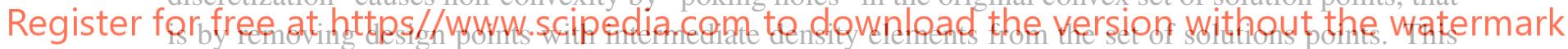

concept is illustrated in Figure 1a. Nonetheless, domain discretization remedies the lack of closedness

of the solution set, simply by limiting the available options of distributing a certain amount of material within a discretized domain.

To alleviate the non-convexity triggered by domain discretization, "relaxation" has to be introduced. Relaxation in this context refers to expanding the solution space to include the potential solution/design points eliminated by domain discretization (in one form or another). In topology optimization, two main relaxation techniques exist; (i) homogenization using optimal ${ }^{3}$ microstructures such as rank-2 laminates, and (ii) non-penalized material interpolation methods which is the variable thickness sheet problem. Even though relaxation creates a well-posed problem, it is a double-edged approach. For instance, consider the ideal case of relaxation introduced by using optimal microstructures such as rank-2 laminates.

\footnotetext{
${ }^{2}$ Although the main domain discretization technique typically employed in topology optimization problems is the finite element method, other techniques have been successfully adopted such as the finite cell method in [19]. The discussion in this section should apply to any domain discretization technique without loss of generality.

${ }^{3}$ Optimal in the sense that the microstructure is capable of attaining the optimal energy bounds; that is utilizing a certain amount of material to the fullest.
} 
On the one hand, relaxation restores the convexity and produces the closest solution possible to the original continuum problem (for specific displacement and density fields). On the other hand, it also means that the topological features of the optimal design are inherently exhibited on the micro scale, not on the macro scale. One way to think about domain discretization combined with relaxation is as follows; (i) the original problem in the continuum formulation consisted of a solution space of infinite dimensions $R^{\infty}$ with discrete design variable $\{0,1\}$ for each dimension, (ii) domain discretization with relaxation transformed the problem into a solution space of finite dimensions $R^{N}$ with continuous design variables $[0,1]$ for each dimension. The beauty of the transformed formulation is that it's numerically bounded, albeit still non-practical.

Given its simplicity of numerical implementation compared to the homogenized rank-2 laminates, from this point forward, our focus in discussion would be on the variable thickness sheet problem without loss of generality. Even though the variable thickness sheet problem results in a nonpractical solution, it's still of some theoretical value. The popularity of the variable thickness sheet problem stems from two characteristics: (i) the fact that it is considered as the maximum bound on structural efficiency for a certain domain discretization. In other words, material utilization is maximized as the solution space is expanded with the freedom of utilizing intermediate density elements. Once the solution space is restricted to enforce feasible discrete $0 / 1$ solutions, the maximum structural efficiency achievable starts to decline, and (ii) it can be used to obtain the global relaxed optimum which is the best candidate to be used as the initial guess towards discrete topology optimization.

In the following section, we discuss the different forms of penalization in use and how it affects the shape

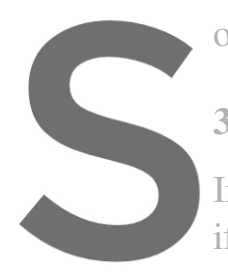
of the objective function

\section{PENALIZATION}

In a topology optimizatio ifying the objective funct
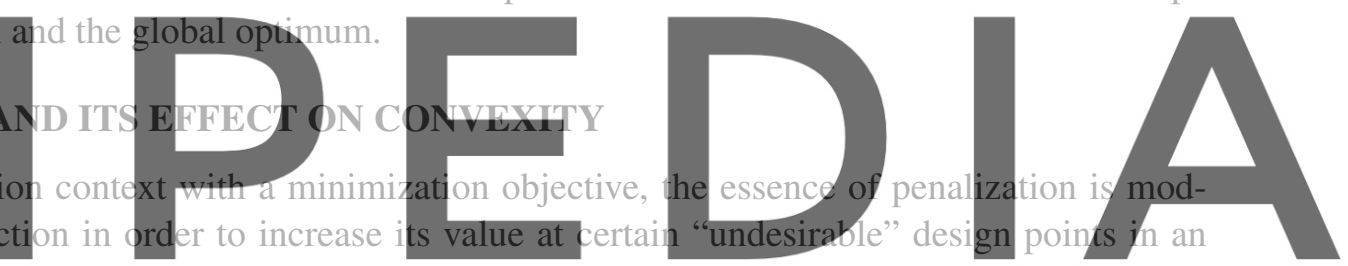

effort to force the local minima towards the remaining unaffected "desirable" design points. In topology

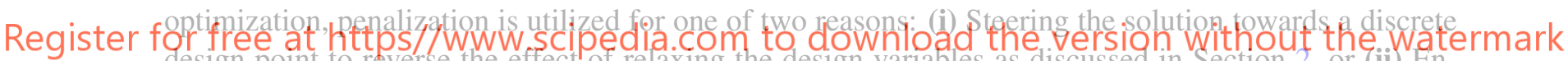
design point to reverse the effect of relaxing the design variables as discussed in Section 2 , or (ii) En- $^{-}$ forcing a design constraint such as minimum/maximum length scales (e.g. manufacturability), and under this category also falls dealing with numerical instabilities such as checkerboarding, mesh dependency, and one-node hinges.

\subsection{Penalization of Intermediate Density Elements}

Penalizing intermediate density elements can be implemented in a number of different forms, they can be categorized as follows:

Implicit - in which the penalization factor is not explicitly recognized or controlled. This type of penalization is achieved using homogenization with a sub-optimal microstructure (i.e. partial relaxation), that is a microstructure that is incapable of obtaining the optimal energy bounds for intermediate density elements, hence penalization is inferred. A prominent example of this category is found in [4] where rectangular elements with rectangular holes were utilized (c.f. [5] for a comprehensive review). Historically, to the best of the authors' knowledge, this form of penalization was the precursor to modern density-based methods, as in the pioneering work by Bendsøe and Kikuchi [4] which was followed by 
the first appearance of the SIMP method in the seminal work by Bendsøe [3].

Explicit - in which the penalization factor can be explicitly recognized and controlled, which is probably the more intuitive approach. This category includes two main approaches; (i) Local; where the material property of interest (e.g. stiffness in a structural mechanics problem, permeability in a fluid flow problem, or conductivity in a heat transfer problem) is penalized explicitly within the local stiffness matrix of each element. This approach is more popular and is the basis of all density-based methods, and (ii) Global; in which the objective function itself is appended with a penalty term that is only active for the undesirable intermediate density elements, and inactive otherwise.

Referring to our discussion on convex functions at the beginning of Section 2, penalization introduces non-convexity to the problem by transforming the objective function's epigraph from a convex set into a non-convex one. The effect of penalization on an originally convex objective function can be visualized in Figure 1b. The value of the objective function at the desirable points (represented by the tick marks on the $\mathrm{x}$-axis) remains unchanged, while it increases at the undesirable points (represented by the values in-between the tick marks).

\subsection{Penalization for Enforcing Design Constraints}

In this subsection, we discuss in detail a few techniques that belong, or at least are relevant, to penalization for enforcing design constraints, and their effect on the problem's convexity. A summary of the Lagrangian multipliers method for enforcing constraints is in order first.

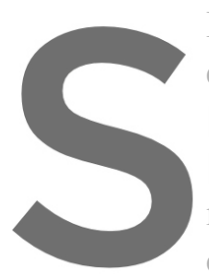
In most, if not all, the soiv enforced using the Lagrant [6] where the only constraint is [29], CONLIN [13], IPOP mented $^{4}$. It's commonly constrained optimization
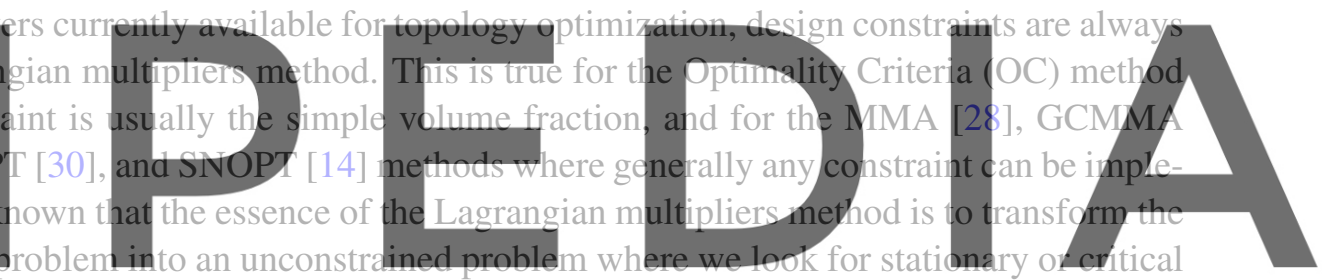

points. Depending on the characteristics of the penalty term, any mathematical constraint added to the

\subsubsection{Volume Fraction Constraint}

Compliance minimization problems must feature a volume fraction constraint. It's usually formulated as a linear summation of elemental densities weighted by their corresponding elemental volumes. Even though the volume fraction constraint is typically implemented as an inequality, such form is only necessary for ease of mathematical implementation and to account for any minor variations due to the discrete nature of the desired final solution. Due to the physical nature of the compliance minimization problem and assuming a well-tuned mathematical algorithm, this constraint is almost always active in all iterations. Hence, without loss of generality, we can limit our discussion to the equality form. In addition, to only consider the potential non-convexification effects of this constraint, let's assume that the original problem has a convex set of feasible solutions, that is the relaxed form of the discretized problem (e.g. a variable thickness sheet problem). It's a known fact that the only equality constraint to produce a convex

\footnotetext{
${ }^{4}$ Unless explicitly noted, any reference to "solvers" in this article is intended to mean the mathematical solvers used to solve the optimization problem itself (e.g. OC, MMA, GCMMA, etc.) and not mathematical solvers of systems of equations (e.g. CG, GMRES, MINRES, etc.).
} 
set is a linear one [10, p. 289]. Such a linear constraint would transform the feasible set of solutions from the $R^{N}$ space to a hyperplane in $R^{N-1}$ space (which is always convex), where $N$ is the number of finite elements. Hence, although the volume fraction constraint is enforced using Lagrangian multipliers, it actually doesn't introduce non-convexification to the problem. This is mainly due to its "linear equality" nature, which results in a still convex set of feasible solutions.

\subsubsection{Other Constraints Enforced through Appending the Objective Function}

In this subsection, we direct our attention to more sophisticated design constraints enforced through appending a penalty term to the objective function, either through Lagrangian multipliers or through an explicit penalty term as in Haber et al. [17, p. 268].

A careful look at the nature of the design constraints enforced through penalty functions or Lagrangian multipliers should provide some insight into the nature of the non-convexification imposed on the objective function as a result. By nature, in this context, we mean the locality vs. globality of the design constraint. With some abuse of terminology, locality vs. globality of the constraint could be loosely described as the extent to which the enforced design constraints affect the individual design variables.

To further elaborate, on one end of the spectrum, a perfect example of an extremely local constraint is the discreteness of the design variables ${ }^{5}$. Such a constraint dictates specific requirements on each design variable; that is each and every design variable has to take a certain value from an extremely limited set $\{0,1\}$. Without taking such extremely local measures, the discreteness constraint cannot be satisfied.

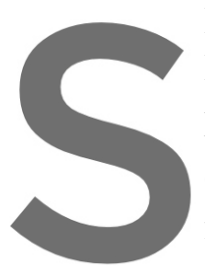
Hence, such local constra In other words, the object point without coming acro On the other end of the fraction constraint. Such
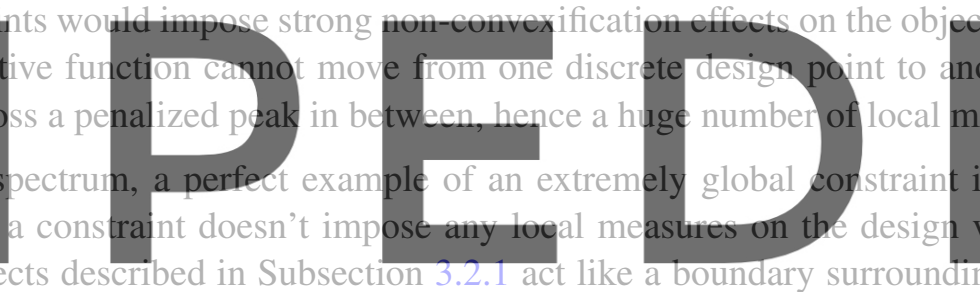

stead, its penalization effects described in Subsection 3.2.1 act like a boundary surrounding an internal feasible solution space (i.e. the hyperplane in $R^{N-1}$ ) that is still a convex set. Hence, any combination of

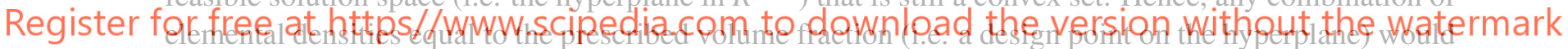
satisfy the constraint, without any local restrictions on the design variables.

This discussion could also be extended to stress-based design constraints. Due to the local nature of the stress constraints, they introduce heavy non-convexification to the objective function. Global measures to overcome the locality problem typically reduce the non-convexification effects, albeit at the cost of not completely satisfying the constraints [18]. A noteworthy remark is that the above "locality vs. globality" discussion is inherently related to the physics of the problem, and consequently would always apply without any dependence on the mathematical solver utilized.

\subsubsection{Mesh-Independency Filtering}

Since the first appearance of mesh-independency filters (i.e. sensitivity filtering by Sigmund [22, 23]), they have become extremely popular in the research community for their effectiveness, computational efficiency, and ease of implementation. It seems prevalent that almost all the work published on black/white

\footnotetext{
${ }^{5}$ Technically, explicit global penalization of intermediate density elements can be thought of as a type of constraint imposed through using the Lagrangian multipliers method.
} 


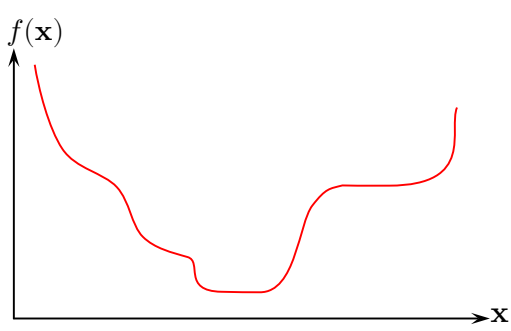

(a)

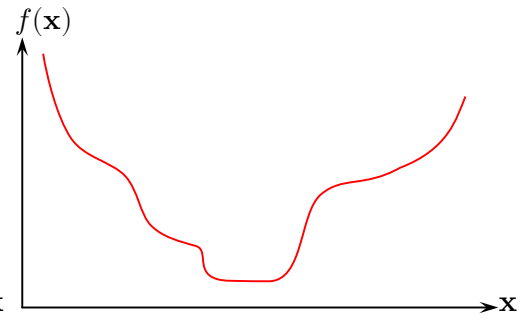

(b)

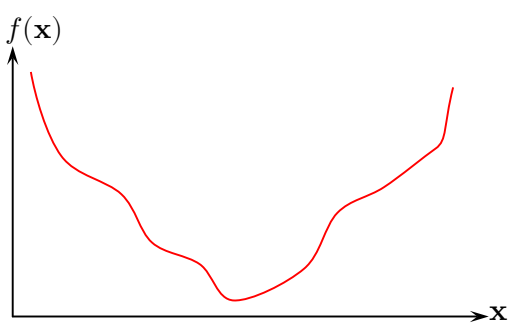

(c)

Figure 2: Generalization of convex functions: a. quasiconvex, b. strictly quasiconvex, and c. strongly quasiconvex. Notice the difference between the stationary points in the three types.

enforcing filters recommends using continuation methods with the filtering schemes (c.f. Sigmund [24, p. 409], Guest et al. [16, p. 249], and Guest [15, p. 128]). In other words, there is a consensus in the literature that sophisticated filtering schemes (i.e. filters that perform any task beyond the simple prevention of checkerboarding and mesh dependency), if implemented without continuation, would generally cause either unstable behavior at worst, or cause convergence to local minima at best. Yet, little attention is usually given to attempting to investigate the effects of the filtering schemes on the convexity of the problem. A probable cause for this general attitude is the fact that the severe localized penalization imposed by density-based methods on intermediate density elements is generally much stronger and tends to overshadow any other form of non-convexification caused by additional constraints.

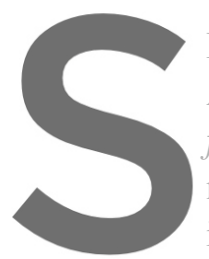

In the following, we attem

At this point, it's worthw

functions. Following the

nonempty convex set in $R$

is true:
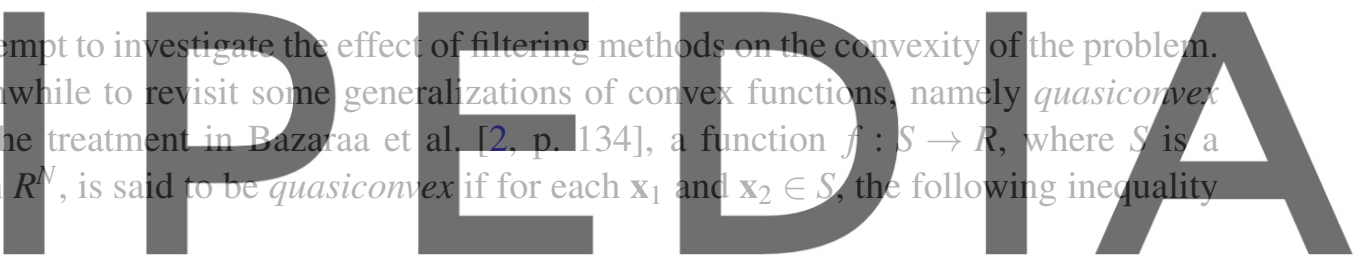

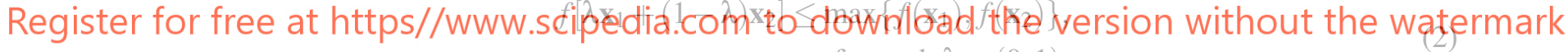 for each $\lambda \in(0,1)$.}

Although quasiconvex functions have a single minimum, their definition don't preclude the existence of multiple stationary points (see Fig. 2a), which is detrimental to iterative solvers. Hence, a more useful concept is the strictly quasiconvex functions. A function $f: S \rightarrow R$, where $S$ is a nonempty convex set in $R^{N}$, is said to be strictly quasiconvex if for each $\mathbf{x}_{1}$ and $\mathbf{x}_{2} \in S$ with $f\left(\mathbf{x}_{1}\right) \neq f\left(\mathbf{x}_{2}\right)$, the following inequality is true:

$$
\begin{aligned}
f\left[\lambda \mathbf{x}_{1}+(1-\lambda) \mathbf{x}_{2}\right]< & \max \left\{f\left(\mathbf{x}_{1}\right), f\left(\mathbf{x}_{2}\right)\right\}, \\
& \text { for each } \lambda \in(0,1) .
\end{aligned}
$$

By enforcing $f\left(\mathbf{x}_{1}\right) \neq f\left(\mathbf{x}_{2}\right)$ and the strictness of the inequality, we actively eliminate any stationary points except at the global minimum (see Fig. 2b). If one is interested in a generalization of convex functions that supports uniqueness of solutions, strongly quasiconvex functions might be of interest. A function $f: S \rightarrow R$, where $S$ is a nonempty convex set in $R^{N}$, is said to be strongly quasiconvex if for each $\mathbf{x}_{1}$ and $\mathbf{x}_{2} \in S$, with $\mathbf{x}_{1} \neq \mathbf{x}_{2}$, the following inequality is true: 


$$
\begin{aligned}
f\left[\lambda \mathbf{x}_{1}+(1-\lambda) \mathbf{x}_{2}\right]< & \max \left\{f\left(\mathbf{x}_{1}\right), f\left(\mathbf{x}_{2}\right)\right\}, \\
& \text { for each } \lambda \in(0,1) .
\end{aligned}
$$

By enforcing $\mathbf{x}_{1} \neq \mathbf{x}_{2}$, strongly quasiconvex functions (see Fig. 2c) assert uniqueness of the global optimum. However, a major disadvantage of the above mentioned functions is that they must have convex lower level sets. That is, for $S$ a nonempty convex set in $R^{N}$, the lower level sets defined as:

$$
L_{\alpha}=\{x \in S \mid f(x) \leq \alpha\} .
$$

for $\alpha \in R$, must be convex [8, p. 95]. This disadvantageous property is usually hard to prove for multivariate functions and doesn't add significant value for an iterative solver. Hence, it would be useful to explore a more generalized concept, that is unimodal functions. Bazaraa et al. [2, p. 156] define univariate unimodal functions as follows; a function $f: S \rightarrow R$, where $S$ is some interval on $R$, is unimodal on $S$ if there exists an $x^{*} \in S$ at which $f$ attains a minimum and $f$ is nondecreasing on the interval $\left\{x \in S: x^{*} \leq x\right\}$ and nonincreasing on the interval $\left\{x \in S: x \leq x^{*}\right\}$. As for multivariate unimodal functions, a number of conflicting definitions exist in literature, specially in the field of Probability and Statistics $[12,20,11]$. Some authors associate the definition of unimodality with that of quasiconvexity as in having convex lower level sets, which is true for univariate functions but not for multivariate ones. In this work, for the definition of multivariate unimodal functions, the natural extension of univariate unimodal functions is utilized [11, p. 38]. That is; a multivariate function $f(\mathbf{x})$ is called unimodal if it's nondecreasing along rays emanating from its global minimum in all directions (see Fig. 3). To preclude the existence of myltiple stationary points, we in every direction emanating fron Returning to our discussion on the effect of filtering on convexity, it distorted. However, unimodal functions are an example of nonminimum attainable through iterative solvers. Hence, it's safe to assume that as long as the modified

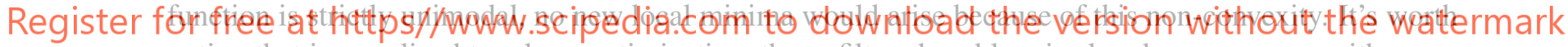
noting that in penalized topology optimization, the unfiltered problem is already non-convex with many local minima. Hence, the above discussion would be only applicable to smaller locally convex parts of the large non-convex function (i.e. a localized valley that has a single minimum). In the following, we reflect this discussion onto sensitivity filtering.

\section{$i$ - Sensitivity Filtering}

Any filtering method starts by determining the neighbourhood of each element; that is the set $N_{e}$ consisting of the elements with centers spatially located within a given filter radius $r$ of the center of element $e$ as follows:

$$
N_{e}=\left\{i \mid\left\|\mathbf{x}_{i}-\mathbf{x}_{e}\right\| \leq r\right\} .
$$

where $\mathbf{x}_{i}$ denotes the spatial location of the center of element $i$.

A modified version of the sensitivity filter that accounts for non-regular meshes with varying elemental 


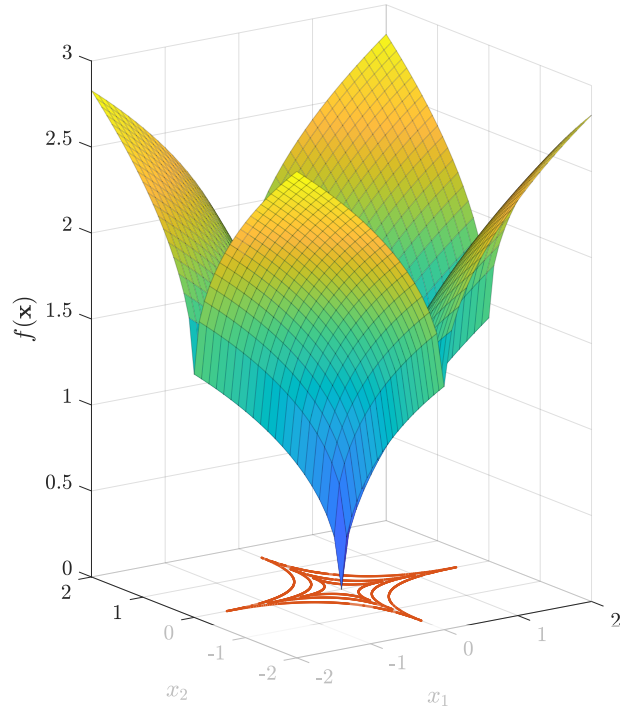

Figure 3: Function $f\left(x_{1}, x_{2}\right)=\sqrt{\left|x_{1}\right|}+\sqrt{\left|x_{2}\right|}$ is an example of a multivariate unimodal function that doesn't belong to any category of quasiconvex functions since its lower level sets aren't convex. Yet, it has a unique global minimum that can be found using iterative solvers.

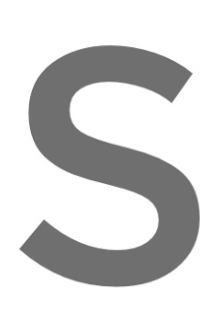

volumes is as follows [
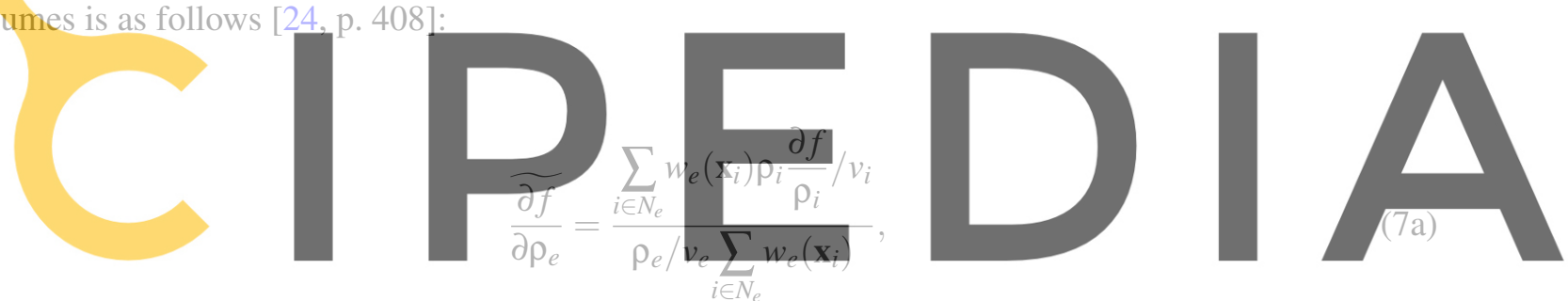

\section{Register for free at https//www.scipedia.Gom to dow}

where $f$ is the objective function, $\rho_{e}$ is the density of element $e, w_{e}\left(\mathbf{x}_{i}\right)$ is a lineariy decaying (coneshaped) weighting function ${ }^{6}$ of element $i$ within the neighbourhood of element $e, v_{i}$ is the volume of element $i$, and $\partial f / \partial \rho_{i}$ and $\widetilde{\partial f / \partial \rho_{i}}$ denote the original and modified sensitivity respectively of the objective function with respect to element $i$.

Our investigative approach for sensitivity filters would be focused on the input to the mathematical solvers; that is whether the filtered sensitivities still constitutes an ascent direction or not. In other words, whether the modified function is locally strictly unimodal or not. In mathematical terms, it means satisfying the following condition:

$$
\frac{\partial f}{\partial \rho} \cdot \frac{\widetilde{\partial f}}{\partial \rho}>0
$$

\footnotetext{
${ }^{6}$ The upcoming proof still applies to other weighting functions such as the Gaussian (bell-shaped) distribution and the constant weighting functions since they are always positive.
} 
In order to ensure that such a dot product is always positive, we need to ensure that each multiplication term is actually positive on its own as follows:

$$
\frac{\sum_{i \in N_{e}} w_{e}\left(\mathbf{x}_{i}\right) \rho_{i} \frac{\partial f}{\rho_{i}} / v_{i}}{\rho_{e} / v_{e} \sum_{i \in N_{e}} w_{e}\left(\mathbf{x}_{i}\right)} \cdot \frac{\partial f}{\partial \rho_{e}}>0
$$

Since $w_{e}\left(\mathbf{x}_{i}\right), \rho_{i}$, and $v_{i}$ are always positive, any multiplicative combination of these quantities would always be positive. Given the fact that sensitivities are always negative in compliance minimization problems, this means that the modified sensitivities are always negative. Hence, each individual term in Eq. 9 is in fact positive as it's a multiplication of two negative quantities. This concludes the proof that the filtered sensitivities in compliance minimization problems always constitute an ascent direction (i.e. the modified function is locally strictly unimodal).

\section{ii - Basic Density Filtering}

This category includes types of density filtering that enforce a grey transition region along the boundaries. Density filtering works by mapping each design point to another design point based on the details of the density filtering scheme. This mapping results in two main effects; (i) a jump from the original design point to the filtered one, and (ii) based on this jump, the sensitivity has to be modified. A typical density filtering takes the following form $[9,7]$ :
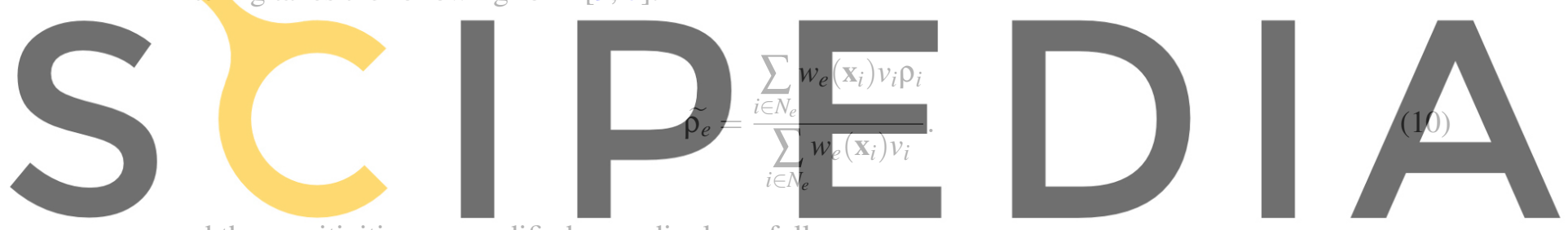

and the sensitivities are modified accordingly as follows:

\section{Register for free at https//www.scipedia.com to download the version without the watermark}

$$
\begin{gathered}
\frac{\partial f}{\partial \rho_{e}}=\sum_{i \in N_{e}} \frac{\partial f}{\partial \widetilde{\rho}_{i}} \frac{\partial \widetilde{\rho_{i}}}{\partial \rho_{e}}, \\
\frac{\partial \widetilde{\rho_{i}}}{\partial \rho_{e}}=\frac{w_{e}\left(\mathbf{x}_{e}\right) v_{e}}{\sum_{j \in N_{i}} w_{e}\left(\mathbf{x}_{j}\right) v_{j}} .
\end{gathered}
$$

Let's focus on the resulting sensitivities first, it's clear that $\partial \widetilde{\rho}_{i} / \partial \rho_{e}$ is always positive since it's a multiplicative combination of $w_{e}\left(\mathbf{x}_{e}\right)$ and $v_{e}$ which are always positive. Hence, according to Eq. 8, the modified sensitivities (Eq. 11a) didn't change signs and still in fact constitute an ascent direction. This proof, unlike the sensitivity filtering proof, applies to any problem, not just to compliance minimization.

As for the modified densities, it's worthy to investigate whether the filter constitutes an affine transformation or not. A careful look at the modified densities (Eq. 10) reveals that it can be put in the form:

$$
\{\widetilde{\boldsymbol{\rho}}\}=[\mathbf{A}]\{\boldsymbol{\rho}\} .
$$


where $\mathbf{A}$ is an $N \times N$ square matrix since $\widetilde{\boldsymbol{\rho}}$ and $\boldsymbol{\rho}$ have the same dimension $N$. This relation constitutes a linear mapping (or an affine mapping to be more general [8, p. 79]) from the original to the modified densities. A rather nice property of affine transformations is that the resultant set is convex if the original is, i.e. the modified densities set is convex if the original densities set is convex, which it is. Relating to our discussion on convex functions in Section 2, this concludes that the modified domain is indeed convex. As for the convexity of the codomain, since the objective function is merely evaluated at a new design point, its codomain undergoes no changes and retains its original convexity, if any existed.

Although we have proven the basic density filter maintains convexity, an intriguing question remains; does the affine mapping exclude any design points from the modified domain? In order to answer this question, we need to investigate whether the matrix $\mathbf{A}$ is invertible or not. This is mainly because a non-invertible affine mapping collapses the space along some directions, i.e. the modified set is smaller than the original one. The matrix $\mathbf{A}$ is singular if its determinant is zero, which can happen in one of two cases (or both): (i) a whole row/column is zero, or (ii) two rows/columns are linearly dependent. The first case cannot happen since the worst case scenario, i.e. having a filtering radius $r$ that doesn't span any adjacent elements, will result in an identity matrix. Any increase in the filtering radius $r$ would fill in more zero elements and hence it's impossible for $\mathbf{A}$ to have a whole row/column of zeros. As for the second case, the only scenario that would result in two (or more) rows/columns being linearly dependent is if two elements have the same neighbourhood of elements $N_{e}$ and a constant weighting function $w_{e}\left(\mathbf{x}_{i}\right)$. The only case we can see this happening is at the free edge of a thin structure comprised of two rows of finite elements, the two elements at the edge would have the same neighbourhood of elements. Of course this is an extremely-unpractical ${ }^{7}$ case and could be safely ignored. Hence, for all practical purposes, the modified domain of a basic density filter doesn't exclude any design points.

\section{iii - Filtering that Enforces 0/1 Designs}

As mentioned before, sensitivity as well as basic density filters enforce a grey transition region along the boundaries. To overcome this issue, a class of filters that enforce $0 / 1$ discrete designs were developed. The first appearance of such filters was in the pioneering work by Guest et al. [16], in which the authors used a nonlinear projection (a regularized Heaviside step function) to ensure a discrete $0 / 1$ boundary and enforce a minimum length scale on the solid phase. A slight modification of this filter could enforce the minimum length scale to the void instead of the solid phase, c.f. Guest [15, p. 125].

Intermediate density penalization, if enforced by the filtering scheme rather than the objective function, would cause unstable behavior (c.f. Guest et al. [16, p. 249] and Sigmund [24, p. 409]). Mainly because the filtering scheme is not designed to seek the minimum discrete point of the problem and consequently its modified sensitivities wouldn't lead to that direction. Hence, it's essential that continuation is utilized in the filtering scheme so as to follow a similar (or lower) degree of penalization as that of the objective function. In what follows, a mathematical justification is presented.

Guest et al. [16, p. 248]'s Heaviside filter takes the following form:

$$
\bar{\rho}_{e}=1-e^{-\beta \widetilde{\rho}_{e}}+\widetilde{\rho}_{e} e^{-\beta} .
$$

where the parameter $\beta$ controls the curvature of the regularization $(\beta=0$ recovers the basic density filter

\footnotetext{
${ }^{7}$ Even with including the elements outside boundary as void elements, the two elements could still have the same neighbourhood of elements (c.f. [24, p. 407] for more details on the treatment of mesh boundaries).
} 
and $\beta \rightarrow \infty$ recovers the Heaviside step function), and $\widetilde{\rho}$ is calculated as in Eq. 10 using a linear weighting function as in Eq. $7 \mathrm{~b}$ (other weighting functions could also be used). The affine mapping argument used in proving the convexity of basic density filtering is not valid here since its converse is not true (i.e. not being an affine mapping doesn't necessarily mean producing a non-convex set). Hence, a different argument is needed, namely the generalization of which the affine mapping argument is a special case.

Soltan [26, p. 114] stated that "a mapping $f: R^{N} \rightarrow R^{M}$ is called convexity-preserving if the $f$-images of all convex sets in $R^{N}$ are convex sets $R^{M}$ ". In our case, $M=N$. Hence, we can focus our attention on a single modified density $\bar{\rho}$, whether its output is a convex set or not. It's clear from the analytical form of the Heaviside filter (recovered as $\beta \rightarrow \infty$ ) that the result contains only two elements $\{0,1\}$. In other words, any combination of neighbourhood elements' weights and densities would always result in a filtered density of 0 or 1 . In other words, certain design points would be excluded from the modified set, and in our case the excluded points are the intermediate density ones. Hence, it's clear that the result is not a convex set, and the filter is not a convexity-preserving mapping. It's worth noting the resemblance between the effect of these filters and the discussion on domain discretization in Section 2.

This concludes our discussion on the convexity of filters, we next move to discussing the various continuation parameters in use.

\section{Conclusions}

Convexity is a core concept in optimization given the abundance of convex programming algorithms and the fact that global optimality can be proven easily in convex problems. However, the current formulation of topology optimization problems inherently introduces non-convexification into the problem. This non-convexification is introduced first by the simple domain discretization required to solve the problem numerically, and later by the intermediate density penalization required to enforce discrete solutions. Various design constraints can be sources of additional non-convexity. In this article, we presented a comprehensive treatment of the various sources of non-convexification introduced into a topology optimization problem. The discretization of the design domain represents a restriction method with a huge pool of local minima. This non-convexity is then remedied through a relaxation of the design variables. In addition, we provided a mathematical treatment on effects of some of the common filtering methods on the convexity of the problem. In conclusion, the non-convexification effects of the penalization of intermediate density elements simply overshadows any other type of non-convexification introduced into the problem, mainly due to its severity and locality. Continuation methods are strongly recommended to overcome the problem of local minima, albeit its step and convergence criteria are left to the user depending on the type of application.

\section{References}

[1] Arora JS, Elwakeil OA, Chahande AI, Hsieh CC (1995) Global optimization methods for engineering applications: a review. Structural Optimization 9(3-4):137-159, DOI :10.1007/BF 01743964

[2] Bazaraa MS, Sherali HD, Shetty CM (2006) Nonlinear Programming. John Wiley \& Sons, Inc., Hoboken, NJ, USA, DOI : 10.1002/0471787779

[3] Bendsøe MP (1989) Optimal shape design as a material distribution problem. Structural Optimization 1(4):193-202, DOI:10.1007/BF01650949 
[4] Bendsøe MP, Kikuchi N (1988) Generating optimal topologies in structural design using a homogenization method. Computer Methods in Applied Mechanics and Engineering 71(2):197-224, DOI : 10.1016/0045-7825(88) 90086-2

[5] Bendsoe MP, Sigmund O (1999) Material interpolation schemes in topology optimization. Archive of Applied Mechanics 69(9-10):635-654, DOI:10.1007/s004190050248

[6] Bendsøe MP, Sigmund O (2004) Topology Optimization: Theory, Methods, and Applications. Springer Berlin Heidelberg, Berlin, Heidelberg, DOI : 10.1007/978-3-662-05086-6

[7] Bourdin B (2001) Filters in topology optimization. International Journal for Numerical Methods in Engineering 50(9):2143-2158, DOI:10.1002/nme.116

[8] Boyd S, Vandenberghe L (2009) Convex Optimization. Cambridge University Press

[9] Bruns TE, Tortorelli DA (2001) Topology optimization of non-linear elastic structures and compliant mechanisms. Computer Methods in Applied Mechanics and Engineering 190(26-27):34433459, DOI:10.1016/S0045-7825(00) 00278-4

[10] Cooper L, Steinberg D (1970) Introduction to Methods of Optimization. W. B. Saunders Company

[11] Dharmadhikari SW, Joag-Dev K (1988) Unimodality, Convexity, and Applications, 1st edn. Academic Press

[12] Dharmadhikari SW, Jogdeo K (1976) Multivariate Unimodality. The Annals of Statistics 4(3):607613

[13] Fleury C (1989) CONLIN: An efficient dual optimizer based on convex approximation concepts. Structural Optimization 1(2):81-89, DOI:10.1007/BF01637664

[14] Gill PE, Murray W, Saunders MA (2005) SNOPT: An SQP Algorithm for Large-Scale Constrained Optimization. SIAM Review 47(1):99-131, DOI:10.1137/S0036144504446096

[15] Guest JK (2009) Topology optimization with multiple phase projection. Computer Methods in Applied Mechanics and Engineering 199(1-4):123-135, DOI:10.1016/J.CMA.2009.09.023

[16] Guest JK, Prévost JH, Belytschko T (2004) Achieving minimum length scale in topology optimization using nodal design variables and projection functions. International Journal for Numerical Methods in Engineering 61(2):238-254, DOI:10.1002/nme.1064

[17] Haber RB, Jog CS, Bendsoe MP (1994) Variable-topology shape optimization with a control on perimeter. In: Proceedings of the 1994 ASME Design Technical Conferences. Part 1 (of 2), ASME, pp 261-272

[18] Le C, Norato J, Bruns T, Ha C, Tortorelli D (2010) Stress-based topology optimization for continua. Structural and Multidisciplinary Optimization 41(4):605-620, DOI:10.1007/ s00158-009-0440-y

[19] Parvizian J, Düster A, Rank E (2012) Topology optimization using the finite cell method. Optimization and Engineering 13(1):57-78, DOI:10.1007/s11081-011-9159-x

[20] Perlman MD (1988) Andrson's Theorem on the Integral of a Symmetric Unimodal Function over a Symmetric Convex Set, and its Applications in Probability and Statistics. Tech. rep., University of Washington 
[21] Petersson J, Sigmund O (1998) Slope constrained topology optimization. International Journal for Numerical Methods in Engineering 41(8):1417-1434, DOI:10.1002/(SICI) 1097-0207 (19980430) 41:8<1417: :AID-NME344>3.0.CO;2-N

[22] Sigmund O (1994) Design of Material Structures using Topology Optimization. PhD Dissertation, Technical University of Denmark

[23] Sigmund O (1997) On the Design of Compliant Mechanisms Using Topology Optimization. Mechanics of Structures and Machines 25(4):493-524, DOI:10.1080/08905459708945415

[24] Sigmund O (2007) Morphology-based black and white filters for topology optimization. Structural and Multidisciplinary Optimization 33(4-5):401-424, DOI:10.1007/s00158-006-0087-x

[25] Sigmund O, Petersson J (1998) Numerical instabilities in topology optimization: A survey on procedures dealing with checkerboards, mesh-dependencies and local minima. Structural Optimization 16(1):68-75, DOI:10.1007/BF01214002

[26] Soltan V (2015) Lectures on Convex Sets, vol 986. World Scientific Hackensack, NJ

[27] Stolpe M (2007) On the reformulation of topology optimization problems as linear or convex quadratic mixed 0-1 programs. Optimization and Engineering 8(2):163-192, DOI:10.1007/ s11081-007-9005-3

[28] Svanberg K (1987) The method of moving asymptotes-a new method for structural optimization. International Journal for Numerical Methods in Engineering 24(2):359-373, DOI:10.1002/nme. 1620240207

[29] Svanberg K (1995) A Globally Convergent Version of MMA without Linesearch. In: Olhoff N, Rozvany G (eds) Proceedings of the First World Congress of Structural and Multidisciplinary Optimization, Pergamon, Goslor, Germany, pp 9-16

[30] Wächter A, Biegler LT (2006) On the implementation of an interior-point filter line-search algorithm for large-scale nonlinear programming. Mathematical Programming 106(1):25-57, DOI : $10.1007 / \mathrm{s} 10107-004-0559-y$ 